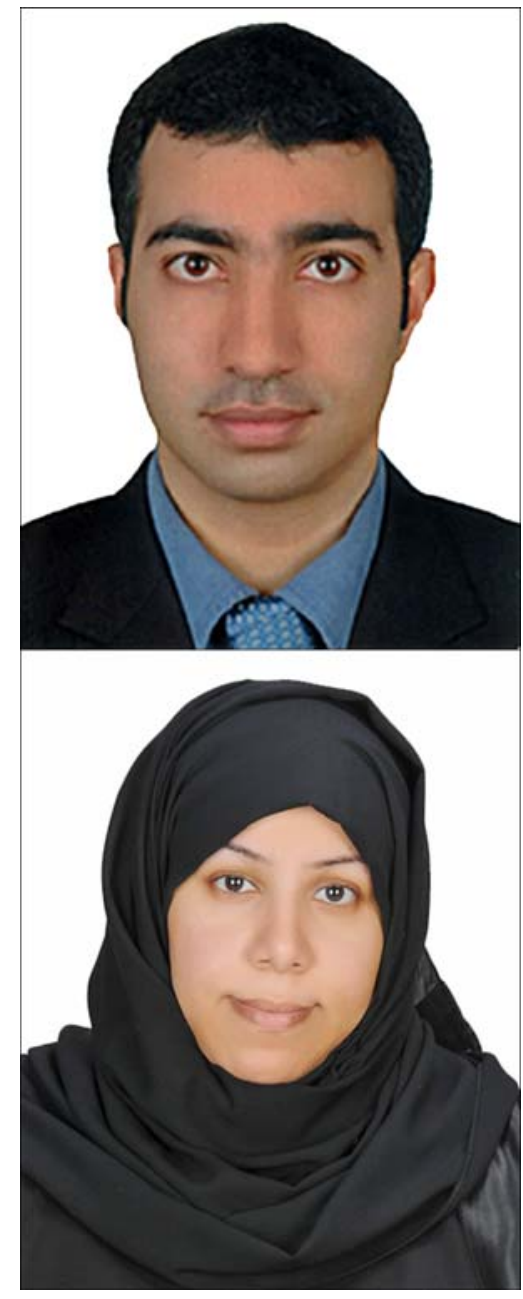

Haitham Jahrami, PhD; Suad Hubail, BSc

\section{Rehabilitation research: Changing focus from outputs to outcomes}

Large amounts of money and significant time and effort are spent on producing rehabilitation research every year. While these resources have contributed to profound advances in rehabilitation, the overall outcomes of the produced research volume are unknown. We still lack a quantification of the added value gained from all of the resources invested in rehabilitation research. In this editorial, we challenge the assumption that research and development without measured outcomes are a good investment. To do this, we must first define inputs, processing, outputs, and outcomes.

Inputs are defined as all the resources that contribute to the production and delivery of outputs. In research, inputs are what we use to conduct research; they are the raw materials, e.g., staff expertise, information and knowledge, ideas, equipment, facilities, assessment, and technology.

Processing refers to the processes or actions that use a number of inputs to produce the desired outputs and ultimately outcomes. In short, processing is what we do to conduct research. For example, writing proposals, testing hypotheses, presenting analyses, sharing results, conducting clinical trials, and reviewing literature are all part of processing.

Outputs are the products created for end users. Outputs may be defined in essence as what we produce from research. Outputs may include conclusions, new knowledge, discoveries, new services, new tests, published papers, conference presentations, principles, and theories.

Outcomes are the medium- to long-term results for specific recipients that come from achieving specific outputs. In essence they are what we achieve from several outputs that add value to the field. In rehabilitation research, outcomes are produced when the recipients (e.g., academics, practicing therapists, students) of research activities accomplish an objective with the outputs. To illustrate, in a rehabilitation department, the outcomes of research activities are achievements such as intervention improvements, cost saving, new services, patient satisfaction, service quality, and patient safety.

Outputs are often measured via publications, peer-review mechanisms, implications of the research, impact factor, and citation frequency. However, measuring outputs is not enough; outcomes should also be measured, and any feedback should be returned to the researchers. The real value of rehabilitation research can only be examined by measuring outcomes. When observing and assessing outcomes, we must also remember that rehabilitation research can also have unintended, unplanned consequences. These consequences need to be identified and monitored so that positive unintended consequences can be used, risks can be managed, and corrective action can be taken for unintended negative outcomes. 
Weiss highlighted three strong benefits of monitoring and measuring outcomes in assessing the effect of medical research efforts. First, measuring outcomes provides a clear and meaningful message regarding the return on investment to funding sources. Second, these outcome assessments serve as a compass to keep research efforts on the right track. Third, the description of research progress in the language of end results highlights the societal good of medical research [1].

Research outputs are often tangible and thus can be measure objectively. Research outcomes are often more difficult to measure and are typically measured subjectively; therefore, rehabilitation researchers ought to search for new ways, techniques, and approaches to measure outcomes [2]. Researchers have the responsibility to plan for their outcomes and should identify areas in which their research projects can produce value.

The world is entering a new era of knowledge and competitiveness, and without a shift in focus, rehabilitation researchers will have little incentive to investigate beyond the outputs of their studies and less understanding of the specific area in which they can help in the application of their work.
Haitham Jahrami, PhD; ${ }^{*}$ Suad Hubail, BSc

Rehabilitation Services, Psychiatric Hospital, Ministry of Health, Kingdom of Bahrain

*Email: hjahrami@health.gov.bh

\section{REFERENCES}

1. Weiss AP. Measuring the impact of medical research: moving from outputs to outcomes. Am J Psychiatry. 2007;164(2):206-14. [PMID:17267781] http://dx.doi.org/10.1176/appi.ajp.164.2.206

2. Westcott R. Outputs and outcomes. Qual Prog. 2008; 41(7):72.

This article and any supplementary material should be cited as follows:

Jahrami H, Hubail S. Rehabilitation research: Changing focus from outputs to outcomes. J Rehabil Res Dev. 2012;49(9):vii-viii.

http://dx.doi.org/10.1682/JRRD.2012.08.0143

ResearcherID: Haitham Jahrami, PhD: A-4436-2012

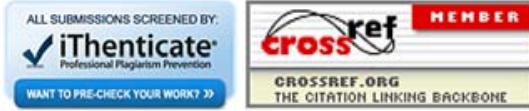

